\title{
Thermal radiation in photonic crystals
}

\author{
Marian Florescu, $, 2, *$ Kurt Busch, ${ }^{3}$ and Jonathan P. Dowling ${ }^{2}$ \\ ${ }^{1}$ Jet Propulsion Laboratory, California Institute of Technology, Pasadena, California 91109, USA \\ ${ }^{2}$ Department of Physics and Astronomy, Louisiana State University, Baton Rouge, Louisiana 70803, USA \\ ${ }^{3}$ Institut für Theoretische Festkörperphysik, Universität Karlsruhe, 76128 Karlsruhe, Germany
}

(Received 20 March 2007; published 17 May 2007)

\begin{abstract}
We analyze the properties of thermal radiation in photonic crystals and show that the spectral energy density, the spectral intensity, and the spectral hemispherical power are only limited by the total number of available photonic states and their propagation characteristics. In addition, we show that the central quantity that determines these thermal radiation characteristics is the area of the isofrequency surfaces and not the photonic density of states as it is generally assumed. Through the presence of partial or complete photonic band gaps and the associated spectral and angular redistribution of photonic states, it is possible to have spectral regions over which the thermal radiation intensity is enhanced relative to the free space blackbody limit and propagation directions along which thermal photon focusing effects appear.
\end{abstract}

DOI: 10.1103/PhysRevB.75.201101

Photonic crystals constitute a new class of dielectric materials in which the basic electromagnetic interaction is controllably altered over certain frequencies and length scales. ${ }^{1}$ In particular, the ability of photonic crystals to significantly alter thermal radiation processes has recently received considerable attention. ${ }^{2-6}$ Besides the scientific relevance, there exists a tremendous potential for technological applications that range from thermophotovoltaic energy conversion devices and light sources ${ }^{2}$ to tunable infrared emitters. ${ }^{7}$ However, nearly all earlier studies fail to recognize the importance of the energy transport velocity in determining the thermal radiation flux and its effects on the temporal redistribution that occurs in the dynamics of thermal radiation in terms of radiation generation and propagation time.

From the standard definition of a blackbody system, ${ }^{8}$ it follows that for any frequency and direction, the flux of the thermal radiation emitted by any material object cannot exceed that emitted by a blackbody at the same temperature and placed in the same environment. This definition is independent of the nature of both the blackbody object and its electromagnetic environment, and does not impose any limit on the amount of thermal radiation emitted by the blackbody. In general, the photonic reservoir of the surrounding medium may support photon modes whose distribution depends on frequency and propagation direction, and the same blackbody object that in free space emits an isotropic thermal flux may inside a photonic crystal emit a thermal flux that is enhanced or reduced relative to Planck's law.

In this paper, we analyze the origin of thermal radiation enhancement and suppression inside photonic crystals, as a prerequisite for the understanding of the thermal radiation properties of finite photonic crystals. Theoretical and experimental studies of the thermal radiation emitted by photonic crystals use the spectral dependence of the photonic density of states (DOS) $)^{2,4}$ or invoke it as a standard reference ${ }^{3,5}$ to interpret their findings. While in simple cases this may lead to correct qualitative predictions, we show that the central quantity that determines the thermal radiation characteristics such as intensity and emissive power is the area of the isofrequency surfaces and not the DOS. Our findings provide a comprehensive understanding of the thermal radiation properties and guidance for future applications.
PACS number(s): 42.70.Qs, 41.20.Jb, 78.66.Vs, 85.60.Jb

We consider a gas of photons in a weakly absorbing dielectric medium with infinitesimal or discrete Bravais-lattice translational symmetry that leads to a dispersion relation $\omega_{\lambda}$ (modes labeled by $\lambda$ ), which is contained in a volume $V$ and is in thermal equilibrium at temperature $T$. The spectral energy density is given by $U(T)=\int_{\omega} d \omega \hbar \omega \bar{n}(\omega, T) \rho(\omega)$, where $\bar{n}(\omega, T)=\left(e^{\hbar \omega / k_{B} T}-1\right)^{-1}$ is the average number of photons with frequency $\omega$, and $\rho(\omega) \equiv 1 / V \Sigma_{\lambda} \delta\left(\omega-\omega_{\lambda}\right)$ denotes the DOS. The spectral energy density is given by $\mathrm{U}(\omega, T)$ $=\hbar \omega \bar{n}(\omega, T) \rho(\omega)$. For a three-dimensional (3D) homogeneous medium (index of refraction $n=\sqrt{\epsilon}$ and dispersion relation $\left.\omega_{\mathbf{k}}=c / n|\mathbf{k}|\right)$, the thermal radiation is isotropic and unpolarized and the DOS is $\rho_{\mathrm{hd}}(\omega)=n^{3} \omega^{2} / \pi^{2} c^{3}$, such that the homogeneous dielectric radiative energy density is enhanced relative to that of free space: $\bigcup_{\mathrm{hd}}(\omega, T)=n^{3} \mathrm{U}_{\mathrm{fs}}(\omega, T)$, here $\mathrm{U}_{\mathrm{fs}}(\omega, T)$ is the free space thermal energy density according to Planck's law. In a photonic crystal, the plane-wave expansion of the electromagnetic field must be replaced by an expansion into Bloch modes. Then the mode index $\boldsymbol{\lambda}=\{n, \mathbf{k}\}$ represents a composite index where $n$ labels the photonic band and the wave vector $\mathbf{k}$ is confined to the first Brillouin zone (BZ). Accordingly, $\omega_{n, \mathbf{k}}$ represents the photonic band structure. The average spectral energy density is $\mathrm{U}_{\mathrm{pc}}(\omega, T)$ $=\mathrm{U}_{\mathrm{fs}}(\omega, T) \times \rho_{\mathrm{pc}}(\omega) / \rho_{\mathrm{fs}}(\omega)$ and must be evaluated via photonic band-structure computations. The DOS of a photonic crystal can be enhanced for frequencies at a photonic bandedge and other van Hove singularities, ${ }^{9}$ and the radiative energy density may exceed its free-space counterpart as illustrated for a two-dimensional (2D) model photonic crystal in Fig. 1. In the following, this model system (square lattice of dielectric cylinders with $\epsilon=8.41$ and radius $r / a=0.15$ in units of the lattice constant $a$; $E$ polarization) will help to illustrate several general features of thermal radiation in photonic crystals.

We now analyze the flow of thermal radiation in an infinite photonic crystal and evaluate the most practical quantities that characterize a general thermal emitter system, such as the directional and spectral distributions of the thermal radiation intensity and emissive power. ${ }^{8}$ Since each mode that contributes to the thermal flow has its own energy transport velocity, the desired spectral representation in terms of a 

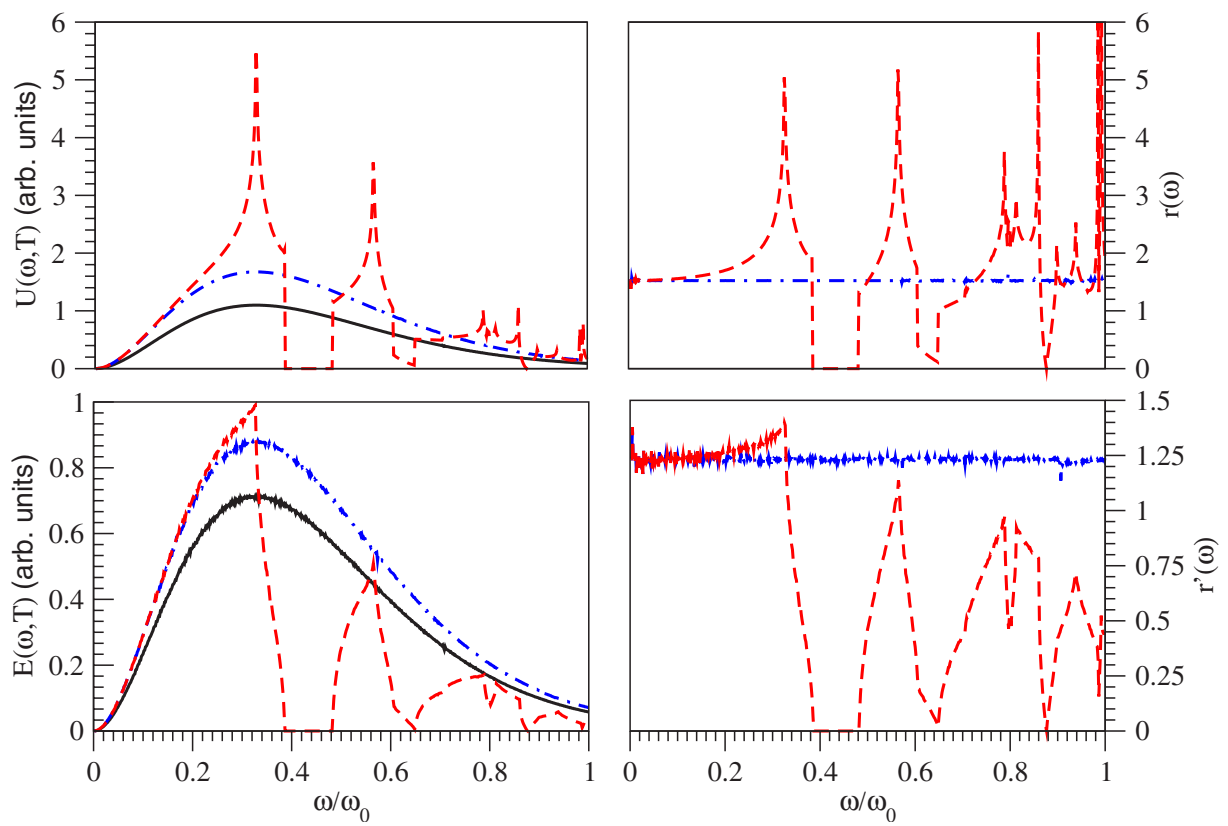

FIG. 1. (Color online) Frequency dependence of the spectral energy density (left-hand panel, upper figure) and spectral hemispherical emissive power (left-hand panel, lower figure) for free space (continous line), a homogeneous dielectric with dielectric constant equal to $\epsilon=1.52$ (dotted-dashed line) and the model photonic crystal (see text; with a long wavelength effective dielectric constant $\left.\epsilon_{\mathrm{av}}=1.52\right)($ dashed line). The temperature is chosen such that the conventional blackbody exhibits a maximum at the frequency of the first van Hove singularity of the model system's DOS, $\omega_{\max } \approx 0.327 \omega_{0}$, and frequencies are measured in units of $\omega_{0}=2 \pi c / a$. On the right-hand panel, we show the ratios of the respective quantities relative to their free space values.

time-averaged Poynting vector becomes more involved. Therefore, we average the flow of electromagnetic radiation over a unit cell and exploit the fact that in structured dielectrics with nondispersive constituent materials, the energy transport velocity coincides with the group velocity $\mathbf{v}_{n, \mathbf{k}}$ $\equiv \nabla_{\mathbf{k}} \omega_{n, \mathbf{k}}$. The spatially averaged current of electromagnetic radiation for a given Bloch mode $\mathbf{J}_{n, \mathbf{k}}(T)$ is then given by $\mathbf{J}_{n, \mathbf{k}}(T)=i_{n, \mathbf{k}}(T) \mathbf{e}_{n, \mathbf{k}}$, where $i_{n, \mathbf{k}}(T)=v_{n, \mathbf{k}}^{(g)} u_{n, \mathbf{k}}(T)$, and $u_{n, \mathbf{k}}(T)$ are the (averaged) $\{n, \mathbf{k}\}$-mode intensity and energy density, respectively. Here, $\mathbf{e}_{n, \mathbf{k}}$ denotes the unit vector along the direction of $\mathbf{v}_{n, \mathbf{k}}^{(g)}$.

Standard quantities that describe thermal radiation are $^{8}$ (i) the directional spectral intensity, $i\left(\omega, \theta_{\mathbf{s}}, \phi_{\mathbf{s}}, T\right)$, defined as the energy emitted per unit time, per unit frequency interval $d \omega$ around $\omega$, per unit elemental projected surface area $d A_{p}$ normal to the direction $\mathbf{s}$, and into a unit solid angle $d \Omega_{\mathrm{s}}$ around the direction $\mathbf{s}$; (ii) the directional spectral emissive power, $e\left(\omega, \theta_{\mathbf{s}}, \phi_{\mathbf{s}}, T\right)=i\left(\omega, \theta_{\mathbf{s}}, \phi_{\mathbf{s}}, T\right) \cos \theta_{\mathbf{s}}$; (iii) the average spectral intensity, $\mathrm{I}(\omega, T)=1 / 4 \pi \int_{0}^{2 \pi} d \phi_{\mathrm{s}} \int_{0}^{\pi / 2} d \theta_{\mathbf{s}}$ $\times \sin \theta_{\mathrm{s}} i\left(\omega, \theta_{\mathrm{s}}, \phi_{\mathrm{s}}, T\right)$; (iv) the hemispherical spectral emissive power, $\mathrm{E}(\omega, \mathbf{s}, T)=\int_{0}^{2 \pi} d \phi_{\mathbf{s}} \int_{0}^{\pi / 2} d \theta_{\mathbf{s}} \sin \theta_{\mathbf{s}} e\left(\omega, \theta_{\mathbf{s}}, \phi_{\mathbf{s}}, T\right)$. For a specific Bloch mode, the corresponding group velocity and $\mathbf{k}_{n}$ vectors may have different orientations, so that the evaluation of the angle-dependent spectral intensity and emissive power in photonic crystals is carried out using the following modal quantities: (i) the directional modeintensity, $i_{n, \mathbf{k}}(T)$, defined as the energy per unit time, per mode $\{n, \mathbf{k}\}$, per unit elemental projected surface area $d A_{p}$ normal to the direction $\mathbf{e}_{n, \mathbf{k}}$ (defined by $\theta_{\mathbf{e}_{n, \mathbf{k}}}, \phi_{\mathbf{e}_{n, \mathbf{k}}}$ ); (ii) the directional mode-emissive power, $e_{n, \mathbf{k}}(T)=i_{n, \mathbf{k}}(T) \cos \theta_{\mathbf{e}_{n, \mathbf{k}}}$. Then, the total intensity and hemispherical emissive power (energy per unit time, into solid $4 \pi$ angle or into the positive s half-space, respectively) are $\mathcal{I}(T)=\Sigma_{n, \mathbf{k}} i_{n, \mathbf{k}}$ and $\mathcal{E}(\mathbf{s}, T)$ $=\sum_{n, \mathbf{k}}^{\prime} e_{n, \mathbf{k}}$, where $\Sigma^{\prime}$ denotes the sum over photonic modes into a hemisphere for which $\mathbf{e}_{n, \mathbf{k}} \cdot \hat{\mathbf{s}} \geqslant 0$. In addition, we have oriented the coordinate axes such that the $z$ axis is along $\mathbf{s}$. In general, the group velocity does not exhibit a simple dependence on the mode energy so that the radiation flux cannot be converted into a frequency integral involving only the total DOS. Instead, we employ an analogy to the calculation of the local DOS (Ref. 9) and write $\mathcal{I}(T)=\Omega_{d} \int d \omega \mathrm{l}(\omega, T)$, $\mathcal{E}(\mathbf{s}, T)=\int d \omega \mathrm{E}(\mathbf{s}, \omega, T)$, where

$$
\begin{aligned}
& \mathrm{I}(\omega, T)=\frac{1}{(2 \pi)^{d}} \frac{1}{\Omega_{d}} \hbar \omega \bar{n}(\omega, T) A\left(\Sigma_{\omega}\right), \\
& \mathrm{E}(\mathbf{s}, \omega, T)=\frac{1}{(2 \pi)^{d}} \hbar \omega \bar{n}(\omega, T) A_{p}^{\prime}\left(\Sigma_{\omega}\right),
\end{aligned}
$$

are the spectral radiation intensity and emissive power, respectively. Here, $\Omega_{d}$ is the solid angle in a $d$-dimensional space. $\quad A\left(\Sigma_{\omega}\right) \equiv \Sigma_{n} A\left[\Sigma_{n}(\omega)\right]=\Sigma_{n} \int_{\Sigma_{n}(\omega)} d S \quad$ and $\quad A_{p}^{\prime}\left(\Sigma_{\omega}\right)$ $\equiv \Sigma_{n} A_{p}^{\prime}\left[\Sigma_{n}(\omega)\right]=\Sigma_{n} \int_{\Sigma_{n}^{\prime}(\omega)} d S \cos \theta_{\mathrm{s}}$ represent the total area of the isofrequency surface $\omega=$ const and the projected areas of the surfaces $\Sigma_{\omega}^{\prime}$ and $\Sigma_{n}^{\prime}(\omega)$ onto a plane normal to the direction s, respectively. $\Sigma_{n}^{\prime}(\omega) \equiv \Sigma_{n}(\mathbf{s}, \omega)$ is the portion of the isofrequency surface $\omega_{n, \mathbf{k}}=\omega$ that lies within the BZ and satisfies $\mathbf{S} \cdot \mathbf{v}_{n, \mathbf{k}} \geqslant 0$.

The photonic DOS that determines the spectral energy density is proportional to the infinitesimal volume in $\mathbf{k}$ space around the isofrequency surface and depends strongly on the group velocities of the modes that lie on this isofrequency surface. In contrast to the DOS, the group velocity does not 


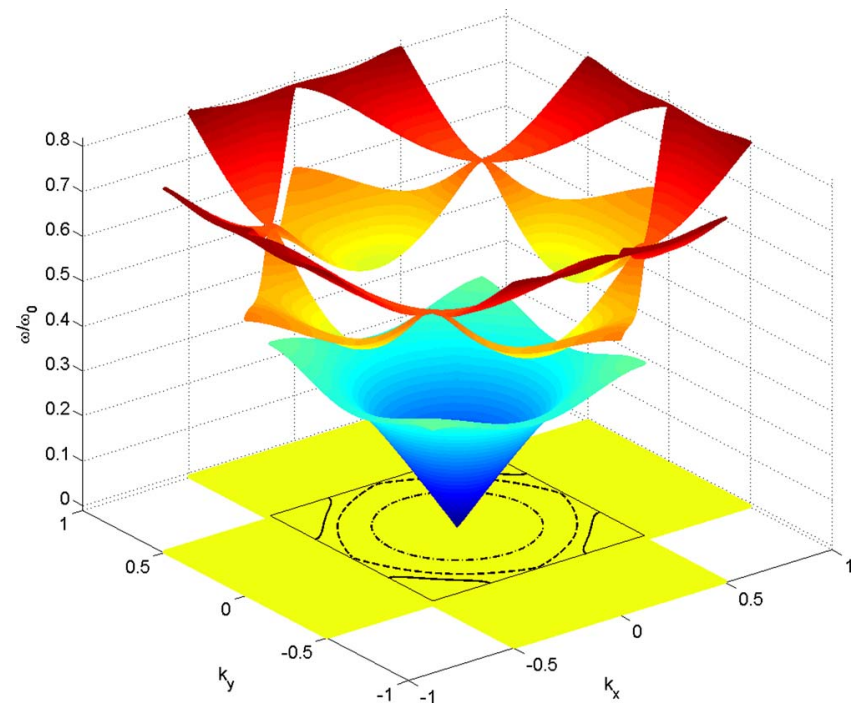

FIG. 2. (Color online) Band structure for a 2D square lattice of dielectric cylinders $(\epsilon=8.41, r / a=0.15)$ in air. In the $\omega=0$ plane we show the k-space domain used (first three Brillouin zones) and the isofrequency contours for $\omega / \omega_{0}=0.25$ (dotted-dashed line), 0.327 (line), 0.345 (continuous line).

contribute to the isofrequency areas, so that the mechanism associated with van Hove singularities (small group velocities) that provides the basis for the strong enhancement of the spectral energy density is absent. In other words, while a photonic crystal may funnel a large energy density into certain spectral regions, the corresponding flow of radiation is usually strongly reduced in exactly those spectral regions due to smaller group velocities. However, we note that the flow of radiation strongly depends on the photonic environment. In the case of a homogeneous 3D dielectric medium, the above-defined spectral intensity and hemispherical emissive power may be expressed as $\mathrm{I}_{\mathrm{hd}}(\omega, T)=\mathrm{I}_{\mathrm{fs}}(\omega, T) \times n^{2}$, $\mathrm{E}_{\mathrm{hd}}(\mathbf{s}, \omega, T)=\mathrm{E}_{\mathrm{hd}}(\mathbf{s}, \omega, T) \times n^{2}, \quad$ where $\quad \mathcal{I}_{\mathrm{fs}}(\omega, T)=\left(\hbar \omega^{3}\right) /$ $\left(4 \pi^{3} c^{2}\right) \bar{n}(\omega, T)$, and $\mathcal{E}_{\mathrm{fs}}(\mathbf{s}, \omega, T)=\left(\hbar \omega^{3}\right) /\left(4 \pi^{2} c^{2}\right) \bar{n}(\omega, T)$ are, respectively, the free space spectral intensity and hemispherical emissive power. ${ }^{8}$ In the case of the photonic crystal, the isofrequency surface areas need to be evaluated numerically. In Fig. 1, we demonstrate that hemispherical emissive power of a blackbody embedded into the 2D model photonic crystal presented above may still exceed that of the same blackbody object in free space. However, this enhancement is smaller than a comparison of the spectral energy densities would suggest and results from a generic feature of photonic crystal dispersion relations (see Fig. 2). For small frequencies, the dispersion relation is essentially linear, corresponding to a homogeneous dielectric with an effective dielectric constant. As the frequency increases, the photonic crystal dispersion relation becomes strongly anisotropic, directional stop bands appear and, ultimately, a complete band gap is opened. The frequency dependence of the isofrequency surface area (contour length, for the $2 \mathrm{D}$ case analyzed here) mirrors this behavior. The directional stop bands open for $\mathbf{k}$ vectors at the boundary of the first Brillouin zone and the associated dispersion relation exhibits a vanishing slope. Exactly at the point where the first directional stop-band appears $\left(\omega / \omega_{0}\right.$ $\approx 0.327$ ), the contour length of the isofrequency surface is maximized (see Fig. 2). For still higher frequencies, the isofrequency contours are topologically disconnected, their length rapidly decreases and vanishes at the photonic band edge. For frequencies above the first fundamental band gap, the isofrequency contours turn into multivalued surfaces, consisting of a set of open contours associated with different bands. Accordingly, the possibility of assembling a maximal length contour is strongly reduced and the values of the surface area remain below that of a homogeneous dielectric.

Next, we determine the angular behavior of the intensity and the hemispherical emissive power by evaluating the corresponding directional quantities. The directional modeintensity and emissive power characterize the flow of thermal radiation in the direction of the corresponding mode's group velocity. The natural angular resolution of the thermal radiation flow is determined by the geometry of the isofrequency surfaces and our approach follows that for dipole radiation in photonic crystals. ${ }^{10}$ For a given direction $\hat{\mathbf{r}} \rightarrow\left\{\theta_{\mathbf{r}}, \phi_{\mathbf{r}}\right\}$, in real space, we define the set $\left\{\mathbf{k}_{n}^{\nu}\right\}_{\nu}$ of stationary $\mathbf{k}$ vectors on the isofrequency surface $\omega_{\mathbf{k}_{n}^{\nu}}=\omega$ for which the group velocity $\mathbf{v}_{\mathbf{k}_{n}}^{\nu}$ is parallel to the direction $\hat{\mathbf{r}}$. Areas and projected areas of isofrequency surfaces can be expressed in terms of a sum over the Gaussian curvatures $\mathcal{K}_{\mathbf{k}_{n}}^{\nu}$ of the stationary points: $\quad A\left(\Sigma_{\omega}\right)=\Sigma_{\nu, n} \int_{\Omega_{d}} d \Omega_{\mathbf{r}} / \mathcal{K}_{\mathbf{k}_{n}}^{\nu}, \quad A_{p}^{\prime}\left(\Sigma_{\omega}\right)$ $=\sum_{\nu, n} \int_{\Omega_{d} / 2} d \Omega_{\mathbf{r}} \cos \theta_{\mathbf{r}} / \mathcal{K}_{\mathbf{k}_{n}}^{\nu}$. The directional spectral intensity and spectral power become independent on DOS and are given by

$$
\begin{gathered}
i\left(\omega, \theta_{\mathbf{r}}, \phi_{\mathbf{r}}, T\right)=\frac{\hbar \omega \bar{n}(\omega, T)}{\Omega_{d}(2 \pi)^{d}} \sum_{\nu} \frac{1}{\mathcal{K}_{\mathbf{k}_{n}}^{\nu}}, \\
e\left(\omega, \theta_{\mathbf{r}}, \phi_{\mathbf{r}}, T\right)=\frac{\hbar \omega \bar{n}(\omega, T)}{(2 \pi)^{d}} \cos \theta_{\mathbf{r}} \sum_{\nu} \frac{1}{\mathcal{K}_{\mathbf{k}_{n}^{\nu}}^{\nu}} .
\end{gathered}
$$

For homogeneous dielectrics, these quantities can be evaluated in closed form and reduce to well-known results. ${ }^{8}$ In photonic crystals they are governed by the topology of the dispersion surface. At long wavelengths, the departure of the isofrequency contour from a homogeneous medium is minimal (see Fig. 2) and the directional spectral emissive power (black curve in Fig. 3) exhibits a Lambertian dependence. As the frequency increases, the thermal radiation becomes more and more anisotropic. Eventually, the directional stop band appear and the isofrequency contours within the first BZ become disconnected. As a result, the isofrequency contours have zero slope normal to the Brillouin zone boundary, and there always exist points of zero curvature along these open isofrequency contours. This implies that the thermal radiation becomes divergent along certain real-space directions (see Fig. 3). This behavior is similar to the focusing of dipole radiation in photonic crystals ${ }^{10}$ as well as phonon focusing in ordinary crystals ${ }^{11}$ and underscores that the spectral and angular redistribution of photonic states play equally important roles in determining the thermal radiation flux. This redistribution is best illustrated in a one-dimensional (1D) photonic crystal, where the spectral energy density depends on the dispersion relation via $\bigcup_{1 d}(\omega, T)=\hbar \omega \bar{n}(\omega, T) /\left[\pi v^{(g)}(\omega)\right]$, 


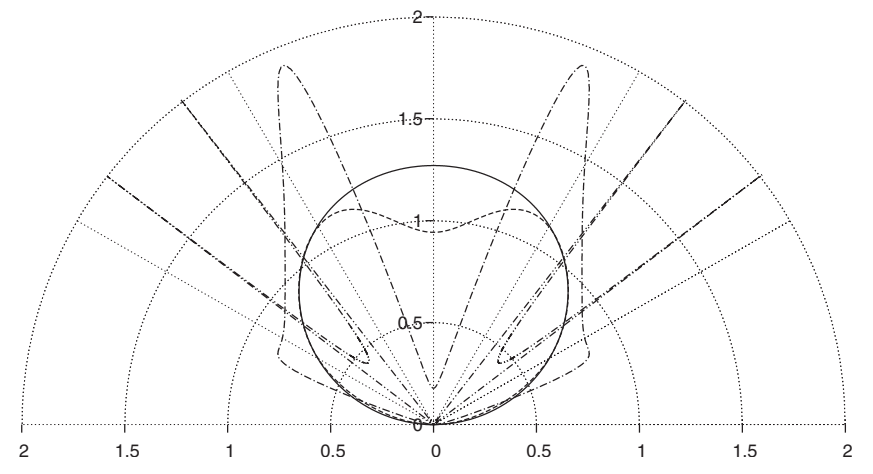

FIG. 3. Dependence of the directional spectral emissive power within the 2D model photonic crystal on the emission angle for $\omega_{1} / \omega_{0}=0.25$ (continous line), $\omega_{2} / \omega_{0}=0.31$ (dashed line), $\omega_{3} / \omega_{0}$ $=0.327$ (dotted-dashed line), and $\omega_{5} / \omega_{0}=0.34$ (double dotteddashed line). For visualization purposes the first three quantities are multiplied by a factor of 3 .

while the spectral intensity and hemispherical power are independent of the photonic dispersion and exhibit an universal dependence on frequency $\mathcal{I}(\omega, T)=\mathcal{E}(\mathbf{s}, \omega, T)$ $=\hbar \omega \bar{n}(\omega, T) / 2 \pi .^{12}$ Despite this cancellation, the presence of a nontrivial dispersion has a profound influence on the dynamics of light-matter interaction. Due to a large local DOS, spontaneous emission can be substantially enhanced and, once emitted, the radiation propagates very slowly. Since in a practical configuration, the atomic system may lose its excitation by transferring it to other degrees of freedom that may be present in the dielectric host (such as phonons), this behavior will be advantageous in minimizing the detrimental effects of nonradiative decay channels. Also, a small group velocity implies a longer interaction of the radiation with the elementary absorbers and enhanced absorption and nonlinear effects can be expected.

In summary, we have analyzed the radiative properties of a photon gas in an infinite photonic crystal in thermal equilibrium and have shown that they exhibit profound departures from the conventional model of a blackbody in free space. For frequencies near van Hove singularities, the spectral energy density as well as the thermal radiation flux may exceed the corresponding quantities for a blackbody in free space. There are two mechanisms contributing to this enhancement. First, similar to a homogeneous dielectric medium, the photonic crystal constitutes an optically denser effective medium than free space. The second mechanism originates from the strong light scattering that leads to a spectral redistribution of the photonic modes. We have shown that the thermal flux must be compounded with an additional factor of the group velocity such that the standard interpretation of thermal radiation characteristics in photonic crystals-and other strongly scattering systems-via the DOS becomes questionable. Moreover, the thermal radiation in photonic crystals shows focusing effects along specific crystalline directions, which may directly impact future applications. Our analysis is relevant for finite photonic crystals as well. In this case, it is necessary to include the transmission and reflection of radiation at the interface between photonic crystal and its surroundings, which can be done following the approach used to analyze the superprism and negative refraction phenomena in photonic crystals. ${ }^{13}$ The reflection at the interface between photonic crystal and free space reduces the thermal flux in a manner consistent with Kirchhoff's law. ${ }^{5,14}$ However, it has been shown that the enhancement of the photonic DOS at the band edge or other spectral singularities may be accompanied by perfect transmission. ${ }^{3}$ As such, the reduction of the thermal flux at these frequencies can only be explained by the competing mechanisms that have been identified by our approach.

Part of this work was performed at the Jet Propulsion Laboratory, California Institute of Technology, under a grant from NASA. Two of the authors (M.F. and J.P.D.) acknowledge support from the Hearne Institute for Theoretical Physics and the LSU LINK, DTO, and ARO. One of the authors (K.B.) acknowledges support by the DFG-Center for Functional Nanostructures of the Deutsche Forschungsgemeinschaft within projects A1.1 and A1.2.
*Electronic address: Marian.Florescu@jpl.nasa.gov

${ }^{1}$ E. Yablonovitch, Phys. Rev. Lett. 58, 2059 (1987); S. John, Phys. Rev. Lett. 58, 2486 (1987).

${ }^{2}$ J. G. Fleming, S.-Y. Lin, I. El-Kady, R. Biswas, and K. M. Ho, Nature (London) 417, 52 (2002); S.-Y. Lin, J. G. Fleming, E. Chow, J. Bur, K. K. Choi, and A. Goldberg, Phys. Rev. B 62, R2243 (2000).

${ }^{3}$ C. M. Cornelius and J. P. Dowling, Phys. Rev. A 59, 4736 (1998); M. Florescu, H. Lee, A. J. Stimpson, and J. Dowling, ibid. 72, 033821 (2005); S. Enoch, Appl. Phys. Lett. 86, 261101 (2005); M. Laroche, R. Carminati, and J.-J. Greffet, Phys. Rev. Lett. 96, 123903 (2006).

${ }^{4}$ C. S. Kee, S. S. Oh, K. J. Chang, J. E. Kim, H. Y. Park, and K. H. Lee, Phys. Rev. B 60, 10573 (1999); Z. Y. Li, ibid. 66, 241103(R) (2002).

${ }^{5}$ C. Luo, A. Narayanaswamy, G. Chen, and J. D. Joannopoulos, Phys. Rev. Lett. 93, 213905 (2004); D. L. C. Chan, M. Soljacic, and J. D. Joannopoulos, Phys. Rev. E 74, 036615 (2006).

${ }^{6}$ S. Y. Lin, J. Moreno, and J. G. Fleming, Appl. Phys. Lett. 83, 380
(2003); S. Y. Lin, J. G. Fleming, and I. El-Kady, Opt. Lett. 28, 1909 (2003).

${ }^{7}$ M. U. Pralle et al., Appl. Phys. Lett. 81, 4685 (2002).

${ }^{8}$ R. Siegel and J. Howell, Thermal Radiation Heat Transfer, 4th ed. (Taylor and Francis, New York, 2002).

${ }^{9}$ K. Busch and S. John, Phys. Rev. E 58, 3896 (1998).

${ }^{10}$ D. N. Chigrin, Phys. Rev. E 70, 056611 (2004).

${ }^{11}$ J. P. Wolfe, Imaging Phonons: Acoustic Wave Propagation in Solids (Cambridge University Press, Cambridge, England, 1998).

${ }^{12}$ J. B. Pendry, J. Phys. A 16, 2161 (1983).

${ }^{13}$ H. Kosaka, T. Kawashima, A. Tomita, M. Notomi, T. Tamamura, T. Sato, and S. Kawakami, Phys. Rev. B 58, R10096 (1998); S. Foteinopoulou, E. N. Economou, and C. M. Soukoulis, Phys. Rev. Lett. 90, 107402 (2003).

${ }^{14}$ S. M. Rytov, Y. A. Kravtsov, and V. I. Tatarskii, Principles of Statistical Radiophysics: Elements of Random Fields (SpringerVerlag, Berlin, 1989); J.-J. Greffet and M. Nieto-Vesperinas, J. Opt. Soc. Am. A 15, 2735 (1998). 